\title{
Meta
}

Journal des traducteurs

Translators' Journal

\section{LÖRSCHER, W. (1991) : Translation Performance, Translation Process, and Translation Strategies. A Psycholinguistic Investigation, Tübingen, Gunter Narr, 307 p.}

\section{Frank G. Königs}

Volume 38, numéro 2, juin 1993

URI : https://id.erudit.org/iderudit/003339ar

DOI : https://doi.org/10.7202/003339ar

Aller au sommaire du numéro

Éditeur(s)

Les Presses de l'Université de Montréal

ISSN

0026-0452 (imprimé)

1492-1421 (numérique)

Découvrir la revue

Citer ce compte rendu

Königs, F. G. (1993). Compte rendu de [LÖRSCHER, W. (1991) : Translation

Performance, Translation Process, and Translation Strategies. A Psycholinguistic Investigation, Tübingen, Gunter Narr, 307 p.] Meta, 38(2), 353-355.

https://doi.org/10.7202/003339ar d'utilisation que vous pouvez consulter en ligne.

https://apropos.erudit.org/fr/usagers/politique-dutilisation/ 
11 LÖRSCHER, W. (1991): Translation Performance, Translation Process, and Translation Strategies. A Psycholinguistic Investigation, Tübingen, Gunter Narr, 307 p.

L'attention portée à des questions d'ordre psycholinguistique concernant l'activité traductrice gagne de plus en plus de terrain, l'objectif des travaux concernés consistant alors à acquérir en la matière des données empiriques relatives à ce qui se passe réellement au cours de cette activité qu'est la traduction, à la façon dont des problèmes de traduction peuvent être surmontés et à la question de savoir quels sont les rapports qu'entretiennent entre eux le processus même de traduction et son résultat, la traduction. La plupart des travaux de recherche dans ce domaine se distinguent par un point commun, à savoir le recours à des données obtenues par des méthodes introspectives pour obtenir les résultats recherchés, données à partir desquelles des déductions peuvent être faites quant aux processus d'opérations mentales mis en ouvre.

Ceci est également le cas du travail qui nous est présenté: l'auteur s'est donné pour objectif de reconstituer des stratégies de traduction en se basant sur des traductions produites oralement — d'ailleurs, les notions de «traduction improvisée» ou même de «tentative d'interprétariat» me sembleraient bien plus adéquates. Les sujets expérimentaux ne sont ni de futurs traducteurs ni des traducteurs professionnels, mais des étudiants allemands anglicistes. Lörscher défend le point de vue selon lequel chaque individu mâ̂trisant deux ou plusieurs langues étrangères effectue automatiquement des opérations mentales analogues à celles entrant en jeu dans la traduction, si bien qu'une stricte démarcation entre traducteurs et apprentis-traducteurs d'une part et les autres apprenants de langues étrangères d'autre part ne semblerait pouvoir mener que partiellement à des résultats différents. Quand bien même dans les rangs des professionnels de la formation des traducteurs on ne cesse de défendre le point de vue selon lequel les traducteurs professionnels et également les apprentis-traducteurs réalisent d'autres démarches mentales que les autres polyglottes, cette prise de position de Lörscher est loin d'être dénuée d'intérêt : cette aptitude qu'est la traduction n'est pas, par définition, étroitement liée à un certain niveau d'aptitude linguistique, pas plus que la performance musicale au piano ni la performance sportive au tennis ne sauraient être le fait exclusif d'Ashkenasy ou d'Edberg ou encore de Seles. Des preuves empiriques permettant de constater des différences effectives quant aux processus mentaux chez les divers groupes de sujets exprérimentaux font encore défaut à l'heure actuelle. Par ailleurs - et cet argument se trouve également mentionné par Lörscher - le degré de réflexes automatiques est si élevé chez les professionnels qu'une investigation permettant de relever des données introspectives s'avérerait bien plus difficile puisque ce type d'approche permettant de recueillir des données consiste à confronter les sujets exprérimentaux à des problèmes qui les contraignent à transposer les connaissances déjà acquises de la mémoire à long terme à la mémoire à court terme afin de pouvoir alors les verbaliser. Je tiens, pour cette raison, la prise de 
position de Lörscher comme étant absolument défendable, et ce jusqu'à preuve - empirique - du contraire.

Une autre hypothèse essentielle de Lörscher consistait à supposer que des traductions produites oralement se prêteraient mieux à livrer des données introspectives que des traductions écrites. Si tel est le cas, Lörscher devrait tout de même tenir compte de l'objection selon laquelle il analyse alors non pas le phénomène de la traduction à proprement parler, mais tout au plus celui de la traduction improvisée, ou mieux encore une forme embryonnaire de l'interprétariat ou de la transposition analogique du sens d'un texte. La situation qui consiste à partir d'un texte écrit pour produire un texte oral représente tant du point de vue du type d'activité que de celui de la fréquence plutôt un cas particulier qu'un cas typique de traduction.

Pour le relevé des données l'auteur a eu recours au procédé consistant à penser tout haut, procédé entre temps fort souvent utilisé dans le domaine de la recherche relative aux sciences de la traduction ( $c f$. par exemple les articles dans House, Blum-Kulka 1986; Faech, Kasper 1987, Séguinot 1989; cf. par ailleurs les travaux de Königs 1986; 1988; 1990 et en préparation; Krings 1986). Il aurait été souhaitable de trouver dans ce contexte une discussion détaillée sur la question à savoir si des introspections orales ne dénaturaient pas dans une certaine mesure la production linguistique orale si bien que des affirmations ou des déductions sur les processus mentaux ne sauraient être faites qu'avec une prudence extrême et en tenant compte d'un certain pourcentage d'erreurs dépassant largement la portion habituelle, proportion sur laquelle Lörscher s'appuie à fort juste titre.

Le corpus des données est important: il comprend au total cinquante-deux traductions effectuées par quarante-huit étudiants anglicistes en première ou en seconde année d'études pour la plupart. Ces traductions étaient pour une moitié des versions anglaises, tandis que l'autre moitié se composait de thèmes anglais. Aucune aide n'était autorisée, ce qui n'est pas sans surprendre; en effet, si l'on considère que la recherche dans le domaine de la psychologie n'a cessé, ces dernières années, pour pouvoir étudier le comportement adopté lors de la solution d'un problème, de plaider de plus en plus en faveur de devoirs à effectuer dans des conditions de travail présentant un maximum d'authenticité et «collant» le plus possible à des situations réelles, on aurait pu s'attendre à une autre décision de la part de Lörscher puisqu'il s'était donné comme objectif l'analyse de la traduction «normale». Pour l'ensemble des neuf textes pris en considération, il s'agit d'une nouvelle, d'un extrait du journal de Max Frisch et d'un texte linguistique sur l'acquisition de l'allemand chez les travailleurs immigrés qui servirent à l'exercice de thème et pour la version de trois articles du Times, d'un texte linguistique relatif à l'histoire de la langue anglaise, d'un texte didactique sur Shakespeare emprunté à un manuel d'anglais / allemand relativement ancien et enfin d'un autre texte didactique sur «l'anglais correct» emprunté à un autre manuel d'enseignement. Le choix des textes constitue un large échantillonnage fort discutable toutefois si l'on y cherche des situations de traduction comportant un certain degré d'authenticité dans lesquelles ces textes seraient susceptibles d'être traduits.

Après un examen minutieux du concept de stratégie et une présentation de l'utilisation des données introspectives, Lörscher décrit dans la partie la plus développée de son travail une multitude de stratégies et de combinaisons de stratégies diverses et ce, tant du point de vue qualitatif (pp. 130-198) que du point de vue quantitatif (pp. 199-264). Le travail aboutit à des conclusions qui constituent des hypothèses et dans lesquelles Lörscher dégage l'orientation que prend le processus chez ses sujets expérimentaux et la tendance de ces derniers à obtenir un maximum de solution à l'aide d'efforts mentaux minimaux. Autant qu'il est possible d'en juger à partir de la présentation de Lörscher, les différents profils de processus des divers sujets expérimentaux sont extrêmement hétérogènes et 
échappent à toute systématisation générale et approfondie; il arrive que, pour quelques cas à problème, l'on puisse reconstituer des processus mentaux naturels présentant des similitudes.

Celui qui veut se pencher sur les questions psycholinguistiques relatives à la traduction ne saurait ignorer le travail de Lörscher. Il est, sur le plan de la description, très minutieux, et il contient, outre des considérations méthodologiques, une analyse des modèles préconisés par les sciences de la traduction traditionnelles, analyse accompagnée d'une prise de position critique fondée. La présentation des diverses stratégies est scrupuleuse et même par moments fastidieuse à la lecture (ce qui est toutefois dans l'ordre des choses). Les données sont interprétées sous divers aspects et livrent d'intéressantes déductions à propos des procédés mentaux développés lors de la traduction improvisée. Pour les chercheurs qui s'intéressent l'empirisme et ceux intéressés par les données concrètes, le travail de Lörscher représente un vrai trésor. Il eût été souhaitable que la conclusion contienne une discussion plus fortement orientée vers la pratique, ce qui, en raison die la richesse des données et des diverses combinaisons de stratégies, aurait pu en résulter cu aurait même pu aboutir à des conclusions sur la didactique de la traduction. Ce travail doit être recommandé à tous ceux qui veulent «se frotter» à cette approche du phénomène de la traduction, car il s'y prête. Ce travail constitue un acquis indiscutable pour les sciences de la traduction parce qu'il présente une quantité non négligeable de données empiriques concrètes et incite à poursuivre la réflexion sur les moyens possibles d'analyser ce qui se passe dans la tête de celui qui est en train de traduire.

FRANK G. KÖNIGS

Ruhr-Universität Bochum, Allemagne

\section{RÉFÉRENCES}

HOUSE, J., BLUM-KULKA, S. (eds.) (1986): Interlingual and Intercultural Communication. Discourse and Cognition in Translation and Second Language Acquisition Studies, Tübingen, Narr.

F.ÆRCH, C., KASPER, G. (eds.) (1987): Introspection in Second Language Research, Clevedon, Multilingual Matters.

KÖNIGS, F. G. (1986): «Der Vorgang des Übersetzens: Theoretische Modelle und praktischer Vollzug. Zum Verhältnis von Theorie und Praxis in der Übersetzungswissenschaft», Lebende Sprachen, 1, pp. 5-12.

KÖNIGS, F. G. (1988): «Auf der Suche nach dem richtigen Wort. Analysen zum lexikalischen Suchverhalten beim Schreiben in der Fremdsprache und beim Hinübersetzen», Fremdsprachen Lehren und Lernen, 17, pp. 99-117.

KÖNIGS, F. G. (1990): «Übersetzen und Schreiben in der Fremdsprache: psycholinguistische Beziehungen und didaktische Konsequenzen», Arntz, R. und Thome, G. (eds.), Übersetzungswissenschaft. Ergebnisse und Perspektiven. Festschrift zum 65. Geburtstag von Wolfram Wilss, Tübingen, Narr, pp. 278-288.

KÖNIGS, F. G. (en préparation): Beim Übersetzen schreibt man - übersetzt man auch beim Schreiben? Ein psycholinguistisch orientierter Vergleich zweier fremdsprachlicher Produktionsprozesse bei fortgeschrittenen deutschen Spanischlernern, Tübingen, Narr.

KRINGS, H. P. (1986): Was in den Köpfen von Übersetzern vorgeht. Eine empirische Untersuchung zur Struktur des Übersetzungsprozesses an fortgeschrittenen Französischlernern, Tübingen, Narr.

SÉGUINOT, C. (ed.) (1989): The Translation Process, Toronto, H. G. Publications.

TIRKKONEN-CONDIT, S. (ed.) (1991): Empirical Research in Translation and Intercultural Studies, Tübingen, Narr. 